\title{
Changing Modalities in the Management of Bronchiectasis after the Invention of Computer Tomogram
}

\author{
Joseph Raj ${ }^{1}$, P. Rani ${ }^{2}$ \\ ${ }^{1}$ Professor of Cardio-Thoracic Surgery, Madurai Medical College, ${ }^{2}$ Assistant Professor, Department of Cardio-Thoracic \\ Surgery, Madurai Medical College, Tamil Nadu, India \\ Corresponding author: Dr. P. Rani M.Ch., Assistant Professor of Cardio-Thoracic Surgery, Madurai Medical College, Tamil \\ Nadu, India
}

DOI: http://dx.doi.org/10.21276/ijcmsr.2019.4.4.29

How to cite this article: Joseph Raj, P. Rani. Changing modalities in the management of bronchiectasis after the invention of computer tomogram. International Journal of Contemporary Medicine Surgery and Radiology. 2019;4(4):D121-D123.

\section{A B S T R A C T}

Introduction: Bronchiectasis can be categorized as a chronic obstructive pulmonary lung disease manifested by airways that are inflamed and easily collapsible, resulting in airflow obstruction with shortness of breath, impaired clearance of secretions often with disabling cough, and occasionally hemoptysis. Bronchiectasis most often presents as a focal process involving a lobe, segment, or sub-segment of the lung, or a diffusing process involving both lungs. Current research aimed to study the role of Computerised Tomogram in the surgical management of Bronchiectasis.

Material and methods: This was a prospective study from 2014 to 2019, a period of 5 years. Total of 22 cases entered in the study. In this study we compare both pre-op CT image and Intra op correlation

Results: CT chest is the confirmatory investigative modality in the diagnosis of bronchiectasis. In 4 patients we plan to do pneumonectomy on the basis of the X-ray chest. After doing CT chest, we changed our idea into lobectomy. Thus, CT is more confirmatory investigations than X-ray chest. With accurate localization and careful surgical management 22 patients with bronchiectasiswere treated effectively.

Conclusion: By careful localization of a lesion and ruling out any lesion in remaining lobes as same site and applying CT scan, proper planning, position of patient during surgery and adopting meticulous safe surgical technique we had gain good results.

Keywords: Bronchiectasis, CT Scan, Pneumonectomy, Lobectomy

\section{INTRODUCTION}

Bronchiectasis is a chronic lung disease caused by a cycle of infection and inflammation that results in permanent structural damage to the small airways and sometimes causes the destruction of adjacent lung parenchyma. Originally described in 1819 by Laennec ${ }^{1}$, the disease was thought to be of waning clinical significance in the 1970s and 1980s. ${ }^{2}$ However, the prevalence of the disease has resurged; in 2005 it was estimated that there were at least 100,000 individuals in the United States who had bronchiectasis unrelated to cystic fibrosis ${ }^{3}$ and a subsequent epidemiologic study has shown a rising prevalence with an annual change of $8.74 \%$ per year. ${ }^{4}$ Bronchiectasis affects people across the spectrum of age but the highest prevalence is in women over the age of 60 years. ${ }^{5}$ Bronchiectasis is also common in other parts of the world; a recent publication from China reported that $1.2 \%$ of individuals over the age of 40 years have been diagnosed with the disease. ${ }^{6}$ Bronchiectasis is a heterogeneous disease; severity is variable and is impacted by the extent of lung involvement, the microbiologic complications and co-existing disorders. Cystic fibrosis is a well-studied cause of bronchiectasis; this review will cover bronchiectasis unrelated to cystic fibrosis (i.e., non-cystic fibrosis bronchiectasis- "bronchiectasis" in this manuscript). Bronchiectasis may be caused by a variety of underlying conditions including genetic abnormalities, immunologic conditions, autoimmune diseases, obstructing airway lesions or chronic aspiration. ${ }^{7}$ It can also be related to pre-existing chronic obstructive pulmonary disease (COPD) or asthma. However, even with intensive investigation for an underlying cause, many cases are thought to be due to prior infections (with or without clear-cut documentation) or are considered to be idiopathic in origin a common complication of bronchiectasis is infection; certain microorganisms have a propensity for causing chronic infection in patients with bronchiectasis. In many cases, the infection occurs and becomes chronic because of the pre-existing lung disease but infections may also be the cause of the bronchiectasis, particularly non-tuberculous mycobacterial infections that cause nodular bronchiectasis. Patients with bronchiectasis are at risk for exacerbations; a recent consensus statement has defined bronchiectasis exacerbations and prevention of exacerbations is an important goal of treatment. Patients with frequent exacerbations are more likely to have decline in lung function and a worse prognosis compared to those who infrequently exacerbate Two tools for assessing prognosis 
in bronchiectasis are the Bronchiectasis Severity Index (BSI) and the FACED score. ${ }^{8}$ Multimorbidity is frequent in bronchiectasis; co-morbidities including malignancy, cognitive impairment, liver disease and cardiovascular disease have a negative impact on outcomes. Current research aimed to study the role of Computerised Tomogram in the surgical management of Bronchiectasis.

\section{MATERIAL AND METHODS}

This was a prospective study from 2014 to 2019, a period of 5 years in Dept of Cardiovascular Thoracic Surgery, Govt Rajaji Hospital Madurai. All the patients underwent surgical excision of the involved lobe without significant morbidity and mortality

Inclusion criteria: Selection of patients done after thorough preoperative investigations with symptoms of bronchiectasis and $\mathrm{CT}$ scan confirmed bronchiectasis patients are taken up for definitive surgery are included in the studies.

Exclusion criteria: Patients without symptoms and patient with associated TB infection, poor candidate for surgery are excluded from the study.

Patients fulfilling the above criteria were enrolled in the study after taking informed consent. A detailed history with special emphasis on symptoms. Chest X-ray, CT thorax, routine hematological, biochemical investigations, and serological test for HIV/ HbsAg were done in all cases. Other investigations included bronchial brushings for cytology, sputum cytology for Acid Fast Bacilli (AFB), pleural fluid analysis.

\section{RESULTS}

Total no patients was 22. The age range affected by bronchiectasis was between 9 to 45 years, with the mean age of 24 years About $64 \%$ of cases were male (14 patients) and $36 \%$ of cases (8 patients) were females, with a male to female ratio of 2:1.4. [Table-1].

Most common site of presentation is Left lung 16 patients

\begin{tabular}{|l|c|}
\hline Total no. of patients & $\mathbf{2 2}$ \\
\hline Male & 14 \\
\hline Female & 8 \\
\hline Age (9 year-45 year) & Mean age 24 years \\
\hline \multicolumn{2}{|c|}{ Table-1: Baseline Characteristic } \\
\hline
\end{tabular}

\begin{tabular}{|l|l|}
\hline Right Lung & Left lung \\
\hline Lower lobe $-1[4.5 \%]$ & Lower lobe $-9[41 \%]$ \\
\hline Entire Rt lung - 2[9\%] & Entire Lt lung - $6[27.5 \%]$ \\
\hline Middle lobe - $2[9 \%]$ & Middle lobe - 0 \\
\hline Upper lobe - $1[4.5 \%]$ & Upper lobe - $1[4.5 \%]$ \\
\hline \multicolumn{2}{|c|}{ Table-2: Site of Involvement } \\
\hline
\end{tabular}

\begin{tabular}{|l|c|}
\hline Procedure done & Percentage \\
\hline Lobectomy & $46 \%$ \\
\hline Pneumonectomy & $36 \%$ \\
\hline segmentectomy & $18 \%$ \\
\hline \multicolumn{2}{|c|}{ Table-3: Type of surgery } \\
\hline
\end{tabular}

[73\%] Right lung involved in 6 patients (27\%). In left lung lower lobe is most commonly involved 9 patients [41\%],entire left lung in 6 patients [27.5\%], upper lobe in 1 patient [4.5\%]. In right lung entire right lung is involved in 2 patients [9\%],middle lobe in 2 patients[9\%],upper lobe in 1 patient [4.5\%],lower lobe in 1 patient[4.5\%].[Table-2]

In our study 8 patients under go pneumonectomy[36\%], 10 undergo lobectomy[46\%],4 patient undergo segmentectomy of lung[18\%] [Table-3].

In 6 patients we plan to do pneumonectomy on the basis of the X-ray chest. After doing CT chest, we changed our idea into lobectomy, and in 4 patients lobectomy is converted into segmentectomy. In advance of CT scan the conversion rate of surgery is nearly $45 \%$.

\section{DISCUSSION}

Surgical resection is considered one of the therapeutic options, for the treatment of bronchiectasis. ${ }^{9-11}$ The reported operative mortality rates are $1-8.6 \%$. Operative morbidity ranges from 14 to $53 \%$, in different series. ${ }^{12}$ In a study conducted in Saudi Arabia to assess the results of surgery for unilateral bronchiectasis, Ashour et al found no operative mortality and $15 \%$ operative morbidity. ${ }^{14}$ The number of patients cured by the operation was $72.5 \%$, with improvement in $27.5 \%$ of patients. This was similar to the numbers reported by others. ${ }^{2}$ Bronchiectasis could be encountered in one or both lungs, single or multiple lobes and many diffuse lung diseases may manifest with bronchiectasis as the primary abnormality. ${ }^{15}$ Izhakian et al., 2016, the right pulmonary bronchiectatic lesions were found to be more in the right middle lobe (25.9\%) than in the right lower lobe (20.7\%), and the left pulmonary bronchiectatic lesions were found to be more in the lower lobe (20.4\%) than that in the upper lobe (20\%). ${ }^{16}$

In our series of 22 cases over a period of 5 years, whom we have operated successfully without significant mortality and morbidity and without residual disease or recurrence. Most of our patients were from low socio-economic group with age group from 9 to 45 years. Males were more than females $2: 1.4$. Bronchiectasis is common in right lung. In my study, I noticed that left lung particularly lower lobe commonly involved. Dry bronchiectasis is more common than wet bronchiectasis. Heamoptysis is the commonest system. With accurate localization and careful surgical management 22 patients with bronchiectasis were treated effectively. Because of commonly adapted left lateral position during sleep in our areas, long length of the left main bronchus and poor knowledge about chest physiotherapy ie. ineffective chest secretions clearance cause left lower lobe bronchiectasis.

\section{CONCLUSION}

$\mathrm{CT}$ chest is the confirmatory investigative modality in the diagnosis of bronchiectasis. Computed tomography particularly high-resolution images are more sensitive and specific for the diagnosis of bronchiectasis. HRCT also helps to identify the anatomical distribution of bronchiectasis which is helpful in diagnosis of any associated condition or cause of bronchiectasis. The Sensitivity and specificity of HRCT are $84-97 \%$ and $82-99 \%$. By careful localization of lesion and ruling out any lesion in remaining lobes as same 
site and applying $\mathrm{CT}$ scan, proper planning, position of patient during surgery and adopting meticulous safe surgical technique we had gain good results.

\section{REFERENCES}

1. Hansell DM. Bronchiectasis. Radiol Clin North Am. 1998;36(1):107-28.

2. Holmes AH, Trotman-Dickenson B, Edwards A, et al. Bronchiectasis in HIV disease. QJ Med. 1992;85(307308):875-82.

3. Ip MS, Lam WK. Bronchiectasis and related disorders. Respirology. 1996;1(2):107-14.

4. Keistinen T, Saynajakangas O, Tuuponen T. Bronchiectasis: an orphan disease with a poorlyunderstood prognosis. Eur Respir J. 1997;10(12):27847.

5. King P, Holdsworth S, Freezer N. Bronchiectasis. Intern Med J. 2006;36(11):729-37.

6. King PT, Holdsworth S, Freezer NJ. Characterisation of the onset and presenting clinical features of adult bronchiectasis. Respir Med. 2006;100(12):2183-9.

7. Koh WJ, Kwon OJ. Bronchiectasis and nontuberculous mycobacterial pulmonary infection. Thorax. 2006;61(5):458; author reply 458.

8. Kolbe J, Wells AU. Bronchiectasis: a neglected cause of respiratory morbidity and mortality. Respirology. 1996; 1(4):221-5.

9. Annest LS, Kratz JM, Crawford FA Jr. Current results of treatment of bronchiectasis. J Thorac Cardiovasc Surg 1982;83(1):546-50.

10. Crutcher RR, Pellegrino ED. Bilateral bronchiectasis. Surgical management with particular attention to the problem of the residual superior segment of the lower lobes. Ann Surg 1960;151(5):715-28.

11. Oscher A, DeBakey M, Decamp PT. Bronchiectasis. Its curative treatment by pulmonary resection. An analysis of ninety-six cases. Surgery 1949;25(3):518-32.

12. Roberts DE, Cole PJ. Use of selective media in bacteriological investigation of patients with chronic suppurative respiratory infection. Lancet 1980;1(2):7967.

13. Roles FC, Todd GS. Bronchiectasis. Diagnosis and prognosis in relation to treatment. Bronchiectasis Med J 1993;2(6):639-43.

14. Ashour M, Al-Kattan KM, Jain SK, Al-Majed S, AlKassimi F, Mobaireek A, et al. Surgery for unilateral bronchiectasis: Results and prognostic factors. Tuber Lung Dis 1996;77(4):168-72.

15. T. Marini, S.K. Hobbs, A. Chaturvedi, K. KaprothJoslinBeyond bronchitis: a 5review of the congenital and acquired abnormalities of the bronchus Insights into Imaging 2017;8(1):141-153

16. S. Izhakian, W.G. Wasser, L. Fuks, B. Vainshelboim, B.D. Fox, O. Fruchter, et al.Lobar distribution in noncystic fibrosis bronchiectasis predicts bacteriologic pathogen treatment. Eur J Clin Microbiol Infect Dis, 2016;35(5):791-796

\section{Source of Support: Nil; Conflict of Interest: None}

Submitted: 21-09-2019; Accepted: 17-10-2019; Published online: 23-12-2019 